\title{
Performance Analysis of an Inductive Contactless Power Transfer System
}

\author{
Yassine Amirat ${ }^{1}$, Gilles Feld ${ }^{1}$ and Yves Auffret ${ }^{1,2}$ \\ ${ }^{1}$ ISEN Yncrea Ouest Brest, France \\ ${ }^{2}$ Céladon Association, Brest, France
}

Email: yassine.amirat@isen-ouest.yncrea.fr and gilles.feld@isen-ouest.yncrea.fr and yves.auffret@isen-ouest.yncrea.fr

\begin{abstract}
Deployment of autonomous underwater vehicle have became a focal point for many industrial application. The main challenge to the autonomous underwater vehicle is the finite mission time during the operation in a harsh environment. The most efficient way to reduce the operational and maintenance costs, and increase the efficiency of the system, the autonomous under water vehicles have to be recharged under water during the operation. This paper proposes an analysis of one side compensation topology and indexes some parameters to be considered when designing an inductive contactless power transfer system for AUV charging.

Keywords-magnetic coupler, contactless power transfer, autonomous underwater vehicle charging.
\end{abstract}

\section{INTRODUCTION}

Contactless power transfer technologies (CPT) has been a focal point in research area and industrial application, this is due to development of mobile transportation and the enhancement of autonomy of such systems. The CPT can be grouped into radiative RF-based charging, generally utilized for farfield transmission, and non-radiative coupling-based utilized for near-field transmission. The far-field power transfers are based on using schemes such as the microwave [1], optical, and acoustic to transfer energy from the transmitter to the receiver. Alternatively, near-field power transfer uses electromagnetic field couplings, such as the inductive, capacitive, and magnetic resonance coupling to transfer energy from the transmitter to the receiver. The concept of inductive wireless power transfer is similar to that of a transformer with a weak coupling, i.e., it uses magnetic field induction as a means to transfer energy between two coils. A primary coil energized by a high-frequency $\mathrm{AC}$ power source is used as a transmitter, while a secondary coil is used as a receiver. Typically, an inductive WPT system consists of three parts, transmitter, inductive coupler, and receiver, as seen in Fig 1. The system can be considered to be static when the distance between the transmitting and receiving coil is constant and dynamic when the distance and the load vary. In spite of the huge knowledge accumulated by several technologies applied in various scientific and industrial fields, the task of contactless power transfer is still an art, for example, for distributed ocean technology such as autonomous underwater vehicles (AUVs), as presented in Fig. 2 operates primarily using the potential energy stored 0005-03)

This work was supported by the ANR MarTERA Project (NO.17 MART-

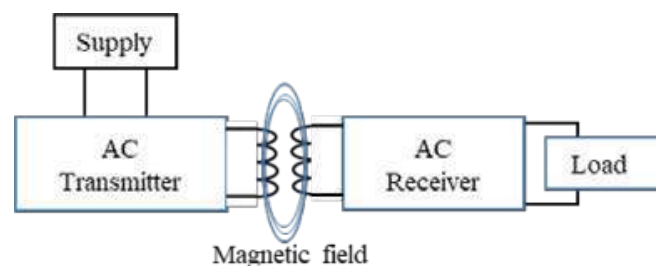

Fig. 1. Inductive power transfer principle.

in batteries to perform oceanic research, search and recovery operations. The batteries carry a finite amount of charge and this state of charge decrease during operation, so they must be charged in order to continue operations. Conventional charging methods involve removal of the AUV from water, and power is provided through a cable connection as illustrated in 3 . Removal of the AUV from the water requires a larger platform with a crew operating it, consequently increasing the operating costs. The main challenge to the autonomous underwater

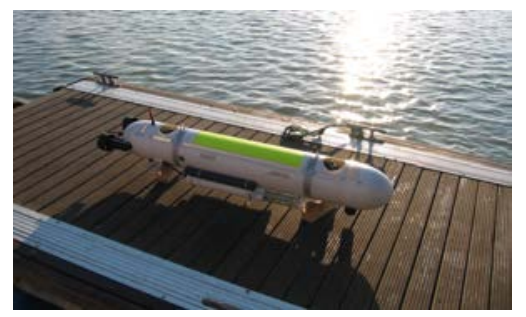

Fig. 2. Modular Autonomous Robot for Environment Sampling [2].
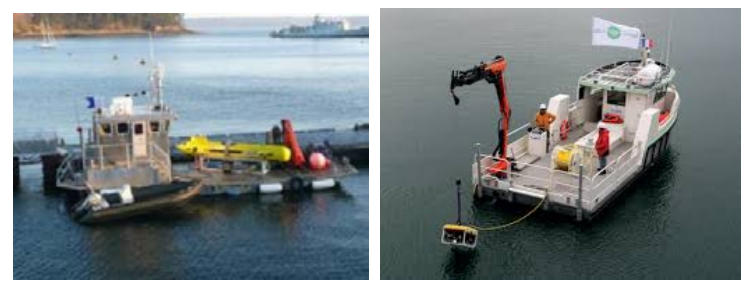

Fig. 3. AUV on the ponton and the "Celadon" ship @Céladon.

vehicle is the finite mission time during the operation which in turn effects with finite capacity of power storage. The power capacity of the autonomous under water vehicles is limited by space constraint of the vehicle and physical distance 
from energy sources. To increase the efficiency of the system, the autonomous under water vehicles have to be recharged under water during the operation. Currently, two techniques are followed to charge the autonomous under water vehicles without extracting them outside the sea. They are electric socket and rotary transformer charging techniques [3], [4]. Unfortunately, both the techniques present drawbacks. In the electric socket configuration, small misalignment may lead to the shorting or corrosion of the conductors. On the other hand, rotary transformer requires bare conductors and high precision for the efficient power transfer. Then the wireless power transfer technique by avoiding the requirement of the direct contact between the charging socket and the load, eliminates the disadvantages of the conventional methods. For this purpose the proposed scheme is illustrated in Fig. 4. The contactless transfer of power to the batteries while the AUV is underwater provides a solution to these operational complications, and increases the independence of the AUV and will prolong operational periods while decreasing maintenance expenditures.

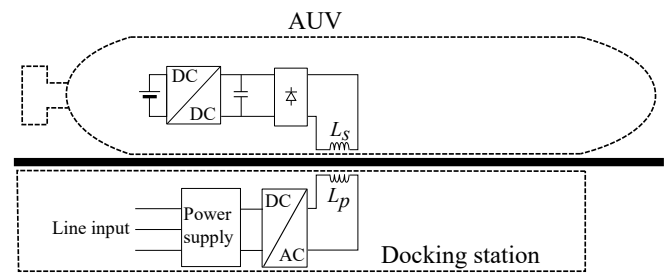

Fig. 4. Docking station for under water charging.

In this paper we propose a topology of a ICPT-based one side compensation, and we present an analysis an assessment of the power transmission performances where the effect of operating distance, operating frequency and the load on the efficiency of the ICPT.

This paper is organized as follows. Section II describes the ICPT principle and enumerates the conventional topologies. In section III the one side compensation based topology is presented, and analyzes the copper losses is carried out through simulation. Section IV presents the experimental validation of the designed prototype and discuss results.

\section{THE INDUCTIVE CONTACTLESS POWER TRANSFER AND TRANSMISSION PERFORMANCE INDICES}

An inductive contactless power transfer (ICPT) system is an electrical system that is typically composed of two electromagnetically coupled transformers with a large air-gap. Because loosely coupled transformers give a low mutual inductance, the system should be driven by a high-frequency power supply to ensure high transfer efficiency. Fig. 5 shows the wellknown ICPT mutual inductance model. The ICPT may suffer considerable power loss due to copper losses, skin effect and eddy currents losses. The loss of power, which is harmful to transmission efficiency, is closely related to the transmission frequency [5].

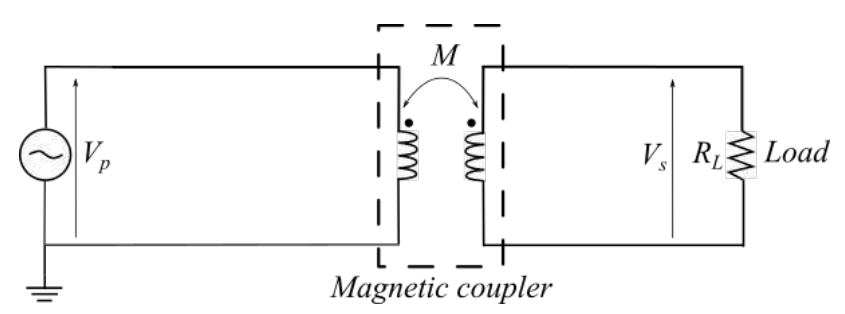

Fig. 5. ICPT mutual inductance model.

Transmission efficiency of an ICPT is related to various factors such as the coupling coefficient of the magnetic coupler, the gap distance, the resonant frequency, the resistance in the circuits and windings, etc. It is also depending on the application environment, in underwater-based applications, both the relative permittivity and the conductivity of the seawater are much higher than those of air. Another aspect that can affect the efficiency and the performances of the ICPT is the compensation topology. Compensation topology was index as is an important aspect when designing an ICPT system, it can enhance the performances of the power transfer by increasing the transmitted active power, and decrease the reactive power rating of the power source [6]. Various literature has presented a plethora of compensation topologies [6]-[8], and the most common are the following four topologies [9]:

- Primary parallel and secondary series (PP-SS)

- Primary series and secondary series (PS-SS)

- Primary parallel and secondary parallel (PP-SP)

- Primary series and secondary parallel (PS-SP)

PS-SS is suitable for low power applications, such as mobile phone charging. PP-SP performs well in very high power applications when the transmission current is generally above 10 A. PS-SP provides a constant current for the load. The big advantage of the PP-SS is that the total input power and the current stress on the MOSFETs can be minimised, which provides a stable voltage for the load. Because stable voltage power transmission is necessary for most of undersea equipment. The details of calculation of the resonant parameters can be found in [9].

\section{PROPOSED TOPOLOGY: ONE-SIDE COMPENSATION}

The proposed scheme is illustrated by Fig. 6 .

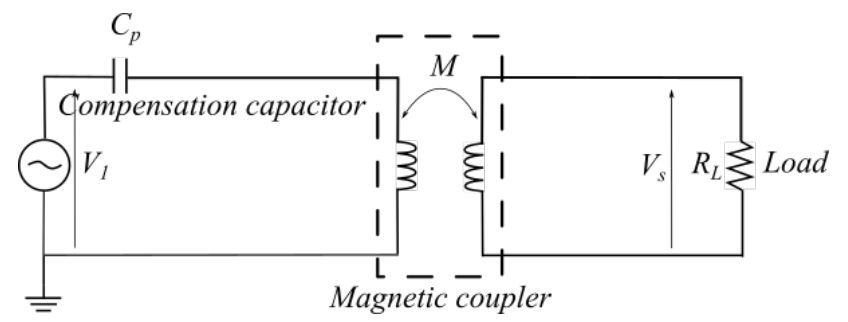

Fig. 6. One side compensation scheme. 


\section{A. Assessment of power losses}

In order to show how to proceed when evaluating the copper losses, the equivalent electrical circuit of the proposed proposed topology is considered as illustrated in Fig. 7. The equivalent mutual voltage circuit model, in which a pair of equivalent voltage sources replaces the primary winding and the secondary winding.

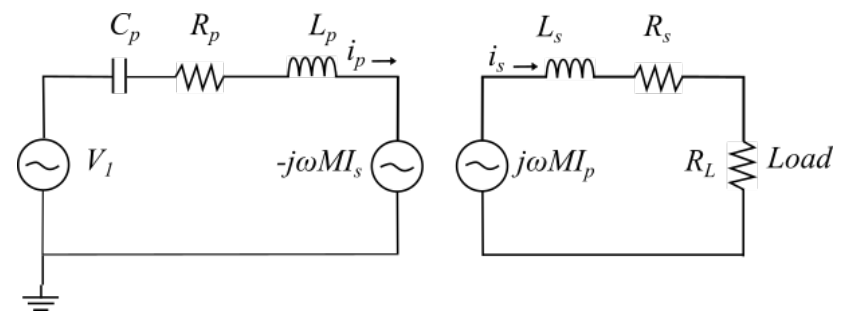

Fig. 7. Equivalent electrical circuit of the one side compensation topology.

According to Kirchhoff's law, and before adding the compensation capacitor $\left(C_{p}\right)$ the model of the circuit can be expressed as:

$$
\begin{aligned}
& V_{p}=R_{p} i_{p}+L_{p} \frac{d i_{p}}{d t}+M \frac{d i_{s}}{d t} \\
& V_{s}=R_{s} i_{p}+L_{s} \frac{d i_{s}}{d t}+M \frac{d i_{p}}{d t}
\end{aligned}
$$

Using the concept of reflected impedance, the electrical equivalent circuit can be taken over to the primary side.

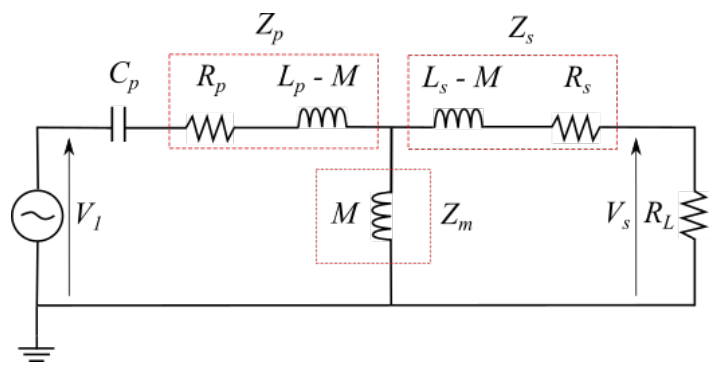

Fig. 8. Simplified electrical scheme.

For a given frequency $f$, (i.e $\omega=2 \pi f$ ) and for a steady state operation:

$$
\begin{gathered}
Z_{p}=R_{p}+j \omega\left(L_{p}-M\right) \\
Z_{s}=R_{s}+j \omega\left(L_{s}-M\right) \\
Z_{M}=j \omega M
\end{gathered}
$$

It will be noted that $Z_{s}$ is the reflected secondary impedance. The equivalent impedance is then:

$$
Z=Z_{p}+Z_{m}\left(Z_{s}+R_{L}\right) \cdot /\left(Z_{m}+\left(Z_{s}+R_{L}\right)\right) ;
$$

At the resonance frequency, the imaginary part of the total impedance must be equal to zero, this can be obtained by adding the compensation capacitor $C$ that satisfy the following expression:

$$
Z_{c}=\frac{1}{C_{p} \omega}=\operatorname{Imag}(Z)
$$

hence,

$$
C_{p}=\frac{1}{\operatorname{Imag}(Z) \omega}
$$

And the set of equation described in

$$
\begin{gathered}
Z_{p c}=R_{p}+j \omega\left(L_{p}-M\right)+{ }^{1} / j . C \omega \\
Z_{s}=R_{s}+j \omega\left(L_{s}-M\right) \\
Z_{M}=j \omega M
\end{gathered}
$$

Adopting the equivalent Thevenin generator theory, we obtain

$$
V_{g t}=V_{1} Z_{m} \frac{V_{1} Z_{m}}{\left(Z_{m}+Z_{p c}\right)}
$$

This yields to,

$$
I_{s}=\frac{V_{g t}}{\left(Z_{g t}+Z_{s}+R_{L}\right)}
$$

Than, The power delivered to secondary side can be expressed as

$$
P_{\text {output }}=R_{L} I_{s}^{2}
$$

and the output voltage is

$$
V_{\text {output }}=R_{L} I_{s}
$$

and finally the efficiency can be expressed as Thus, the power transmission efficiency in air $\eta_{\text {air }}$ is defined as the ratio of the load-consuming power to the total input power, which can be written as

$$
\eta_{\text {air }}=\frac{P_{\text {output }}}{P_{\text {input }}} \times 100 \%
$$

When studying an ICPT, it is also important to have an estimate of the magnetic induction, in order to avoid the saturation phenomenon. For sinusoidal current the maximal magnetic induction $B_{\max }$ is

$$
B_{\max }=\frac{\left|V_{p}\right|}{\left(4.44 N_{p} S_{f} f\right)}
$$

It will be noted that this analysis is valid regardless the type of the electrical topology, and the formulation depends only on the placement of the compensation capacitors $\left(C_{p}\right.$ and/or $C_{s}$ ). To figure out the resonant frequency, the load of ICPT system is varied from 5 to 160 and the frequency from $10 \mathrm{kHz}$ to $100 \mathrm{kHz}$. Figures 9-13 depict the performance of the proposed topology for various frequency and various loads. The effect of the frequency $f$ and the load $R_{L}$ on the efficiency is shown in Fig.14. As the figure shows, although the efficiency always increases as the frequency increases, the curve is totally different when the system is under a different load. The flat surface in Fig.14 shows clearly that the efficiency reaches its high value and remains constant and insensitive to changes in the load and the frequency, which can be achieved when the system operates above a certain frequency and with certain larger loads. When designing an ICPT system, it is important to take care for the admissible values of electrical parameters, such as the voltage across the primary winding $V_{p}$, the magnetic induction, the transmitted 
power and the transmission efficiency. In the above analysis we have considered only resistive losses, for total losses estimation a finite element model was developed and presented in next subsection.

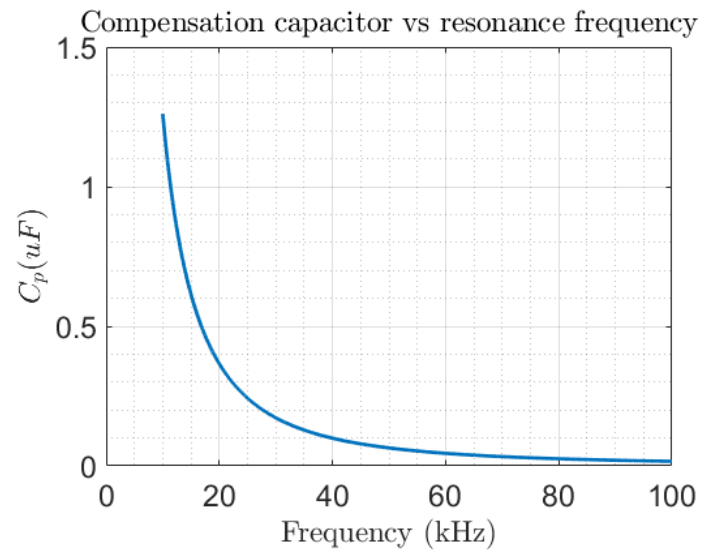

Fig. 9. Resonance capacitor variation versus frequency.

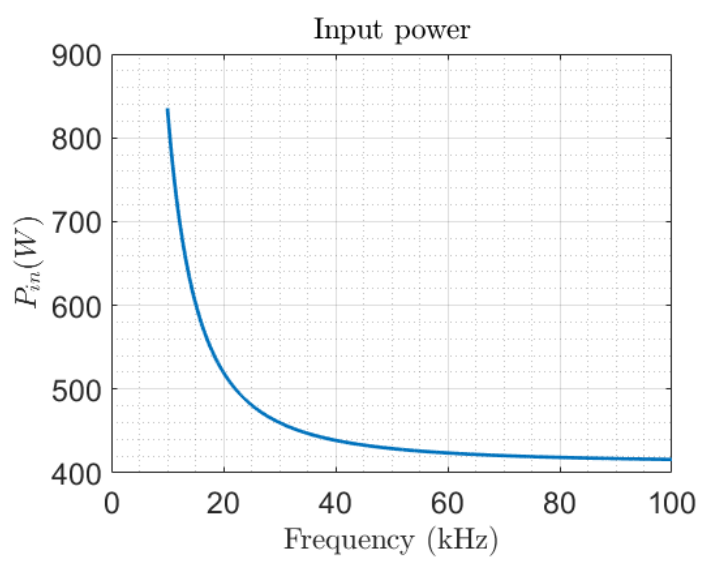

Fig. 10. Input power variation versus frequency.

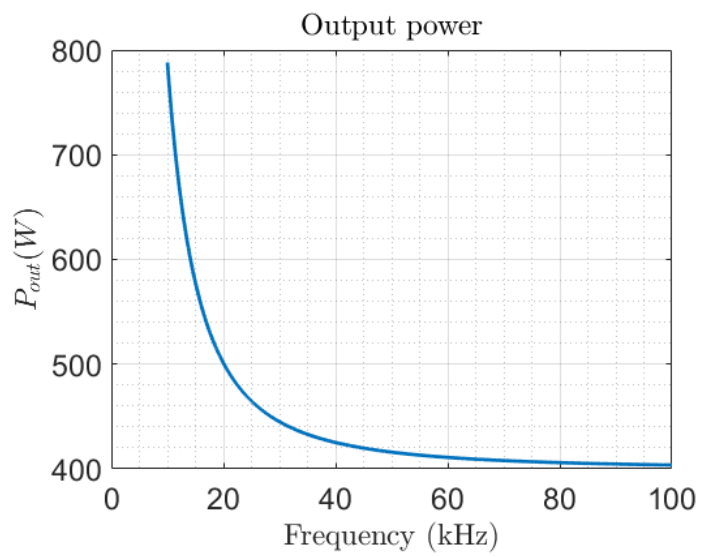

Fig. 11. Output power variation versus frequency.

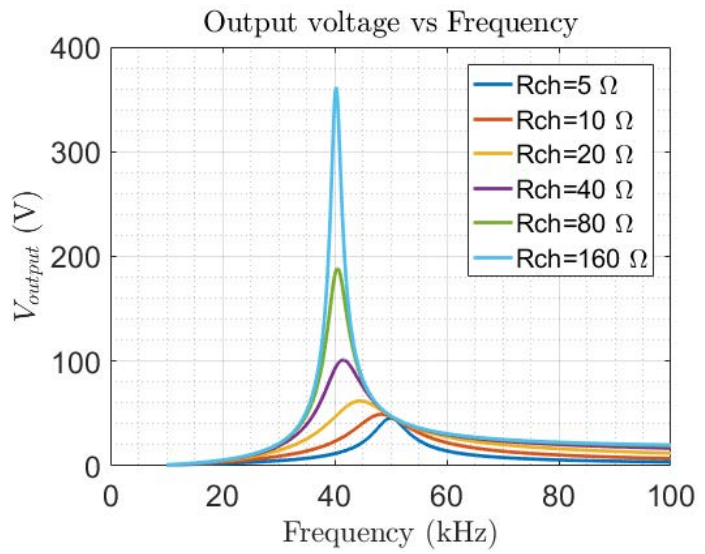

Fig. 12. Out voltage variation versus frequency and resistance load.

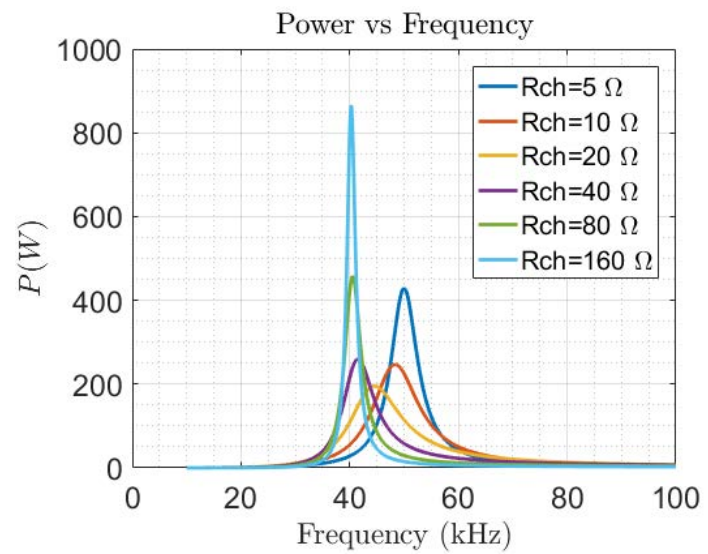

Fig. 13. Output power variation versus frequency and resistance load.

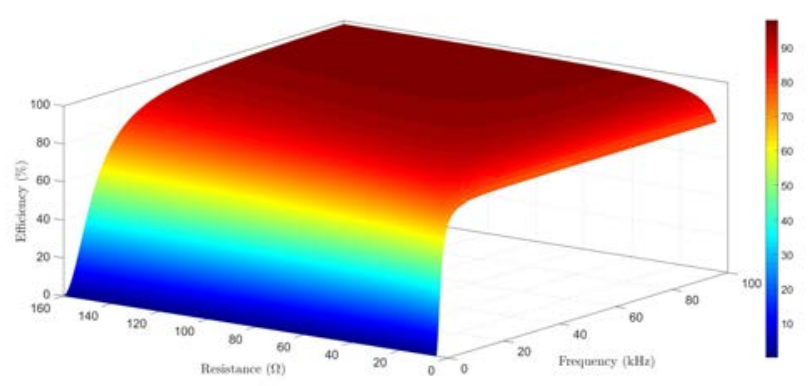

Fig. 14. Transmission efficiency of ICPT in air.

\section{B. Analysis of Finite Element Simulation}

Figure 15 shows an illustration of the structure of the couplers with the ferrite core. Compared with couplers without ferrite cores, this type of coupler has a smaller volume and lower power loss because the smaller radius means that less winding is needed to obtain the same coupling strength. A primary winding (PW) or secondary winding (SW) is fixed around the top of the center pillar of a ferrite core to minimise the leakage inductance. The ICPTs are governed by Ampereś law and Faradayś law among four Maxwell equations, this 


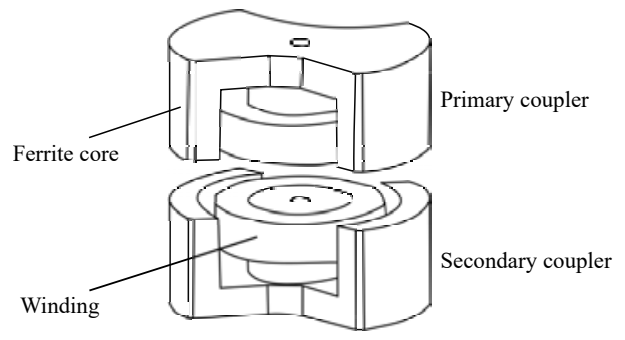

Fig. 15. The structure of the couplers with the ferrite core.

governing equations of ICPTS for sinusoidal magnetic field, voltage and current are approximated as follows:

$$
\begin{gathered}
\nabla \times H=J_{c}+J_{e}, \quad \nabla \times E=-\frac{\partial B}{\partial t}=-j \omega B, \\
\nabla \times B=0, \quad \nabla \times J_{e}=0, \\
B=\mu H, \quad J_{e}=\gamma E,
\end{gathered}
$$

where $J_{c}$ is the excitation current density and $J_{e}$ is the eddy current density. To analyze different the effect of the distance on self inductance $L_{p}$ and $L_{s}$ of the PW and SW respectively, and on the mutual inductance $M$, we have simulated the coupler using finite element software, and the simplified model is illustrated by Fig. 16. First the magnetic coupler divide the coupler space in air (or seawater) into area 1 , area 2 and area 3 , corresponding to subscript $m_{1}, m_{2}, m_{3} . m_{1}$ represents the media between the two halves of magnetic coupler, it can be the air or seawater, $m_{2}$ and $m_{3}$ present the media or the material of the two halves, and is assumed to be a soft magnetic ferrite $(\mathrm{Fe}-\mathrm{Ni}-\mathrm{Zn}-\mathrm{V})$ and The $\mathrm{PW}$ or $\mathrm{SW}$ is located on the boundary of each area. The variable $d$ is the gap distance between the PW and the SW, which is filled with air (or seawater). Figure16 depicts the distribution of the magnetic flux density of the two-dimensional axisymmetric model.

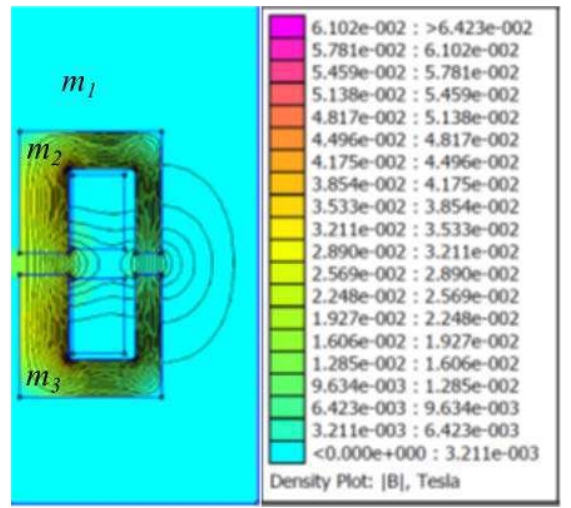

Fig. 16. Flux density of a two-dimensional axisymmetric model.

To figure out the gap between the two halves of the coupler, the distance $d$ is varied from $1 \mathrm{~mm}$ to $5 \mathrm{~mm}$. For a given frequency and a given load (current), and after numerical resolution of the finite element model, the post-processing of the flux density allows us to obtain $L_{p}, L_{s}, M$ and $k$ as depicted in Fig. 17 and Fig. 18 respectively, it is clearly shown that the magnetic linkages coupling the coils are significantly reduced as the distance between PW and SW increases, resulting in fast-dropping mutual inductance, and hence the variation of the gap distance $d$ affects the system parameters such as the resonant frequency, the efficiency and the transmission capacity. Therefore, it is theoretically necessary to maintain a constant gap distance between the primary winding (transmitter) and the secondary winding (receiver).

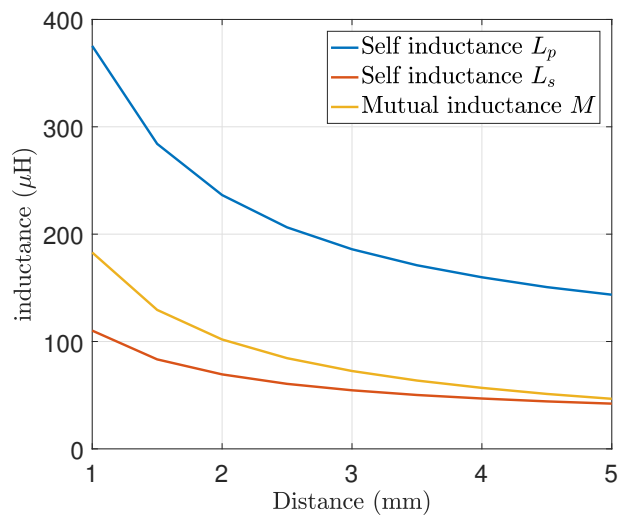

Fig. 17. Self and mutual inductances variation versus distance.

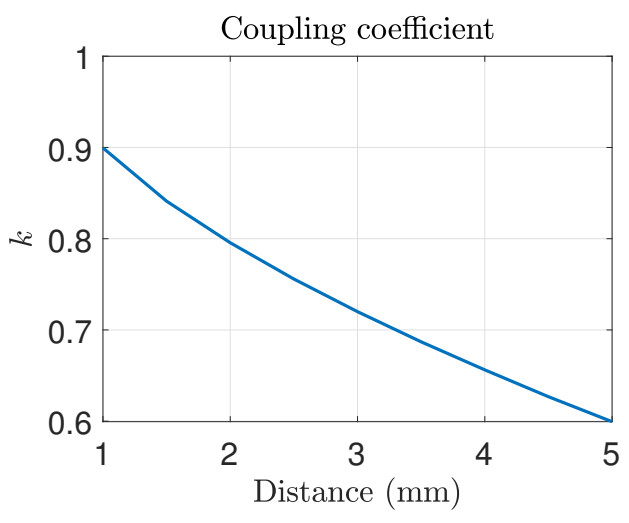

Fig. 18. Coupling coefficient variation versus distance.

To analyze the effect of the winding, a FEM simulation was carried out by assuming that the PW contains 24 turns. As illustrated by Fig. 19, losses are concentrated near the air gap where the flux leakage is high, and this is explicitly indexed by Fig. 20, where losses distribution is presented according the spiral or turn location in $\mathrm{r}, \mathrm{z}$ coordinates for different frequency. This corroborate the fact that the winding have also an impact on the system performance parameters such as efficiency and hence the transmission capacity.

\section{LABORATORY EXPERIMENTS}

Figure 21 shows the layout of the experiment in the laboratory. The primary coupler is driven by a DC supplier with a constant current and a silicon-carbide-based inverter for frequency adjustment. The secondary coupler is linked to 
TABLE I

MEASUREMENT FOR THE EXPERIMENTAL TEST BECH.

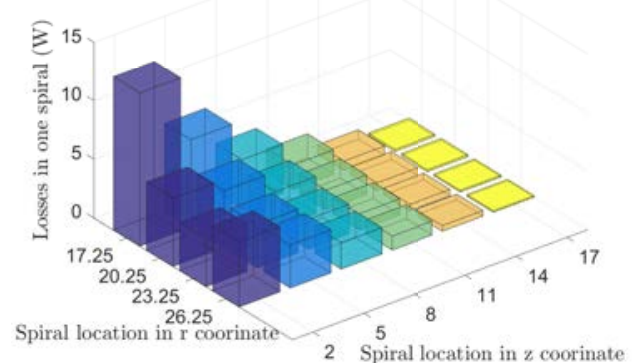

Fig. 19. Losses in on spiral versus its location in $\mathrm{r}, \mathrm{z}$ coordinates for $f=50$ $\mathrm{kHz}$ and a current $I_{p}$ of $10 \mathrm{~A}$.

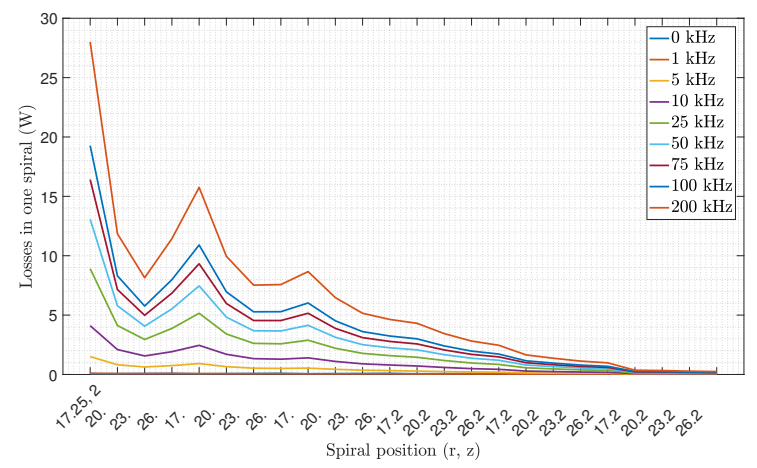

Fig. 20. Losses in on spiral versus its location in $r, z$ coordinates and frequency.

a rectifier with a variable resistance load. The measurement instruments can display the voltage and the current of the load received to calculate the experimental efficiency. The pairs of couplers were made for a resonant frequencies of $50 \mathrm{kHz}$. The couplers are fixed with a gap distance of $5 \mathrm{~mm}$ and either directly exposed to the air. The measured values are presented in table I.

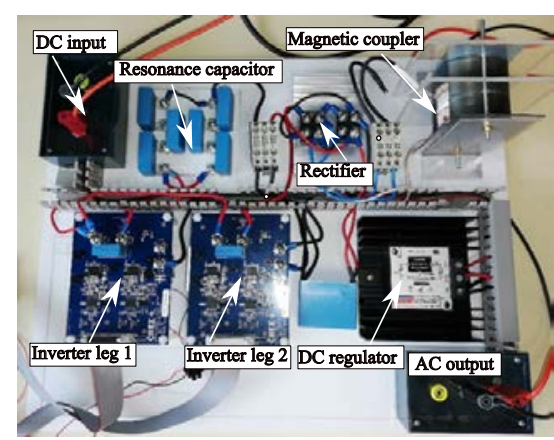

Fig. 21. Experimental setup.

\section{CONCLUSION}

In this paper, an analysis of an inductive contactless power transmission design is analysed. First, the transmission ef-

\begin{tabular}{|c|c|c|}
\hline$V_{d c}$ & $I_{d c}$ & $P_{\text {input }}$ DC side \\
\hline $60 \mathrm{~V}$ & $8.4 \mathrm{~A}$ & $504 \mathrm{~W}$ \\
\hline$P_{\text {input }}$ PW side (inverter side) & $P_{\text {output }} \mathrm{SW}$ side & $P_{\text {output }}$ Rectifier \\
\hline $442 \mathrm{~W}$ & 416 & 402 \\
\hline
\end{tabular}

ficiency of ICPT was derived analytically using a mutual inductance circuit model and by solving Finite element model. By analyzing the effect of the transmission frequency of ICPT on both the efficiency and the power loss. A case study was performed by simulation to determine the optimum transmission frequency for an ICPT system numerically, which was characterized by different loads, and with the proposed compensation topology, simulation results has shown that beyond a certain frequency it is theoretically possible to achieve an efficiency greater than $90 \%$. When designing an inductive contactless power transfer system it is important to take into consideration many parameters and correlating all of them is impractical and provides little insight into the overall design objectives. A laboratory experiment was then conducted to confirm the theoretical analysis and frequency selection. Experiments were carried out firstly in the air, in order to evaluate the behavior of the power electronics components and the ICPT, and assess the transmission performance with different loads. The results confirmed that the designed system was able to contactless transfer power with an average transmission efficiency of $94 \%$ in the magnetic coupler. Further investigation must be carried out in seawater, in order the evaluate the optimum operating distance for a given frequency.

\section{REFERENCES}

[1] W. C. Brown, "The history of power transmission by radio waves," IEEE Transactions on Microwave Theory and Techniques, vol. 32, no. 9, pp. 1230-1242, 1984.

[2] N. A. Cruz and A. C. Matos, "The mares auv, a modular autonomous robot for environment sampling," in OCEANS 2008, 2008, pp. 1-6.

[3] "Underwater mobile docking of autonomous underwater vehicles," in 2012 Oceans, 2012, pp. 1-15.

[4] R. Trevisan and A. Costanzo, "A 1-kw contactless energy transfer system based on a rotary transformer for sealing rollers," IEEE Transactions on Industrial Electronics, vol. 61, no. 11, pp. 6337-6345, 2014.

[5] J. Zhou, D. jun Li, and Y. Chen, "Frequency selection of an inductive contactless power transmission system for ocean observing," Ocean Engineering, vol. 60, pp. 175 - 185, 2013. [Online]. Available: http://www.sciencedirect.com/science/article/pii/S0029801813000085

[6] W. Zhang and C. C. Mi, "Compensation topologies of high-power wireless power transfer systems," IEEE Transactions on Vehicular Technology, vol. 65, no. 6, pp. 4768-4778, 2016.

[7] J. P. C. Smeets, T. T. Overboom, J. W. Jansen, and E. A. Lomonova, "Comparison of position-independent contactless energy transfer systems," IEEE Transactions on Power Electronics, vol. 28, no. 4, pp. 20592067, 2013.

[8] G. A. Covic and J. T. Boys, "Modern trends in inductive power transfer for transportation applications," IEEE Journal of Emerging and Selected Topics in Power Electronics, vol. 1, no. 1, pp. 28-41, 2013.

[9] W. Zhou and H. Ma, "Design considerations of compensation topologies in icpt system," in APEC 07 - Twenty-Second Annual IEEE Applied Power Electronics Conference and Exposition, 2007, pp. 985-990. 